\title{
CCNG1 AND FDXR GENE EXPRESSION LEVELS AFTER RADIATION THERAPY IN BREAST CANCER PATIENTS
}

\section{Trenceva Katerina ${ }^{1}$, Eftimov Aleksandar ${ }^{2}$, Petlichkovski Aleksandar ${ }^{3}$, Jakjovski Zlatko $^{4}$, Topuzovska Sonja ${ }^{5}$}

\author{
${ }^{1}$ Institute of Public Health of the Republic of North Macedonia - Skopje \\ ${ }^{2}$ Insitute of Pathology, Faculty of Medicine, Ss. Cyril and Methodius University in Skopje, R. \\ North Macedonia \\ ${ }^{3}$ Institute of Immunobiology and Human Genetics, Faculty of Medicine, Ss. Cyril and \\ Methodius University in Skopje, R. North Macedonia \\ ${ }^{4}$ Institute of Forensic Medicine, Faculty of Medicine, Ss. Cyril and Methodius University in \\ Skopje, R. North Macedonia \\ ${ }^{5}$ Institute of Medical and Experimental Biochemistry, Faculty of Medicine, Ss. Cyril and \\ Methodius University in Skopje, R. North Macedonia \\ e-mail:ktrenceva@yahoo.com
}

\begin{abstract}
Introduction: Radiation biomarkers represent parameters which, through their quantification, reflect the interaction of the biological system with ionizing radiation (IR) as a physical agent in the environment and are object of study of the biological dosimetry. Of all identified radioresponsive genes that consist "gene signatures" of IR, the gene for ferredoxin reductase, FDXR, is prominent with its promptness and relevance in estimation of exposition to IR. It is also known as adrenodoxin reductase. The gene for CCNG1 represents another in vitro and in vivo validated radiation biomarker involved.

Materials and methods: In this non-randomized, controlled open-trial clinical study, 57 patients with diagnosed breast cancer and 50 healthy individuals were included. Isolation of RNA from $3 \mathrm{~mL}$ peripheral blood and gene expression analysis with qRT-PCR were performed for detection of expression of FDXR and CCNG1 genes.

Results: A statistically significant difference in the threshold cycle was confirmed for FDXR and CCNG1 in the analyzed period between 24 and $48 \mathrm{~h}$ after radiation.

Conclusion: Gene expression is emerging as a highly powerful readout for biodosimetry. Exposure to IR leads to many cellular responses including modification of gene expression. Many genes have been reported to be radiation-responsive at the transcriptional level and monitoring of their expression in blood samples can potentially be used for rapid, minimally invasive high-throughput biological dosimetry purposes.

Keywords: ionizing radiation, breast cancer, FDXR, CCNG1

\section{Introduction}

Radiobiology studies the action of the ionizing radiation (IR) upon living organisms [1]. Radiation biomarkers (BM) represent parameters which, through their quantification, reflect the interaction of the biological system with IR as a physical agent in the environment and are object of study of the biological dosimetry [2].
\end{abstract}


During radiotherapy (RT), the energy of the ionizing rays, directed to a specific goal, can provide cure or palliation of the disease. In breast cancer, RT represents locoregional treatment which can be part of a combined treatment with other therapeutic modalities [3].

One of the ways to achieve therapeutic effect of RT is damage of the DNA molecule of the tumour cells, which has as a consequence significant changes in the transcriptome of the cells involved [4-7]. The set of reactions in the DNA response towards the damage (DDR, DNA- damage response) mediates in reparation of the DNA molecule, the function of the control points all of the cell cycles or the process of apoptosis. One of the DSBs (doublestrand breaks, two-chain interruptions in the DNA molecule) - activated DDR cascades as a result of exposure to IR which represents ATM/CHEK2/p53 signal pathway, in which a large part of the transcription responses are dependent on the tumor-suppressor and the transcription factor p53 [8]. The identification of the molecular biomarkers of IR is useful in the follow-up of the progress of RT, as well as in prediction of the outcome on its very beginning [9]. Encouraged by the development of high-flow technologies for genome profiling, the interest of the researchers lately has been directed towards an analysis of the changes in the levels of gene expression after exposition to IR [10-15]. It is considered that p53 has a key role in the processes involved in the response towards IR [6]. By using PCR (polymerase chain reaction) platforms potential radiation biomarkers have been identified - a group of genes with changed expression, usually up-regulated, under the action of IR, representing the so-called "gene signatures" or "metagenes" of IR [11, 12, 15, 16].

According to Kabacik et al. through application of animal models or via blood samples of patients with RT development of simple clinical tests may be enabled for prediction of the possibility of manifestation of radiotoxicity and introduction of individualized RT [17]. Also, the conclusions from the systematic examination of Lacombe et al. [18] are identical, for confirmation of the current knowledge about the robustness of the "gene signatures" as a relevant tool of biodosimetry.

The study of Macaeva et al. [19] as well as the first and the second RENEB (Running the European Network of Biodosimetry, RENEB) trial [20, 21] simultaneously use different platforms (microarrays and PCR), i.e. conditions for cultivating isolated cells from periferal blood in generating mutually compatible "gene signatures", demonstrating the robustness of the genetic expression as a relevant tool in following the exposure to IR.

Of all identified radioresponsive genes that consist "gene signatures" of IR, the gene for ferredoxin reductase, FDXR, is one of the most prominent with its promptness and relevance in estimation of exposure to IR. It is also known as adrenodoxin reductase [12, 20, 22]. The gene for FDXR codes mitochondrial protein which initiates transport of electrons from NADPH through ferredoxin of cytochrome P450 [23]. It is included in p53-mediated reactions, as well as in ROS (Reactive Oxygen Species) - associated apoptosis [24, 25]. It represents a gene critical for p53-dependent tumor-suppression through iron-regulatory protein 2 (IRP2, iron regulatory protein) [26]. The 46-fold increase of the expression of this gene is noticeable $24 \mathrm{~h}$ after radiation with $4 \mathrm{~Gy}$ of human blood [22]. This high level of expression, in combination with relatively low levels of endogenous expression and interindividual variability, as well as the linear increase of the expression observed at low and high doses of IR make this gene especially attractive for examination of the exposition of this physical agent in the blood.

The CCNG1 gene, which encodes Cyclin G1 protein, represents another in vitro and in vivo validated radiation biomarker in 553 -mediated processes, specifically, in the regulation of the downtime of the cell cycle [20, 23, 31]. Its in vitro basal as well as radiation-induced expression is insignificantly affected by antioxidants, anti-inflammatory agents and bacterial lipopolysaccharide [32]. 
The most frequently used internal control in in vivo studies is the gene for HPRT1 (encodes Hypoxanthine Phosphoribosyltransferase 1 - housekeeping gene) [29, 31, 32]. This was the reason why it was selected for normalization of the levels of the target genes in our study.

In order to make validation of modified expression of radioresponsive genes after exposure to IR with X-rays, we set the following aims of our study:

1. Relative quantification of the level of expression of two genes of interest, FDXR and CCNG1, and one "housekeeping" gene, HPRT1, in samples of peripheral blood from patients exposed to high doses (2Gy/fraction) of X-rays during external RT at three precisely defined time points in the beginning of the radiation treatment of a breast, expressed as fold change of their expression level.

2. Comparison of magnitude of the genetic expression growth of the two radioresponsive genes at three time periods: between the second and the first fraction of RT; between the third and the second fraction of RT and between the third and the first fraction of RT (cumulative effect).

3. Establishment of the curve dose - response towards the ionizing radiation (X-rays) in patients with breast RT for the two target genes in the period between the first and the second time point from blood sampling.

\section{Materials and methods}

\section{Patients, blood sampling and irradiation}

In this non-randomized, controlled open-trial clinical study, 57 patients were included (56 female and 1 male) aged $55.3 \pm 9.8$ with diagnosed breast cancer and referred to elective RT at the University Clinic for Radiotherapy and Oncology in Skopje. Forty of these patients were previously subjected to radical mastectomy with partial or complete dissection of axillary lymph nodes, in 11 - quadrantectomy, while in the other 6 excision of the breast tumor was performed. Patients aged under 25 years were excluded from the study; those who were exposed to IR in the last 10 days prior to the beginning of RT during a diagnostic procedure, if they received concomitant radio- or chemotherapy and/or were with severe comorbidities.

According to the Declaration of Helsinki, $64^{\text {th }}$ World Medical Association, each patient approached the study after receiving the information about the study and by signing an informed consent, approved by the Ethics Committee for research with people at the Faculty of Medicine, UKIM in Skopje.

This study comprised a control group of 50 healthy individuals (aged over 25 years), who were referred to a routine laboratory check-up, without previous chemo- and radiotherapy. They were surveyed in terms of age, status of active smoking, OTC (Over-the-Counter) therapy, symptoms of a cold.

During a regular laboratory routine control, with venipuncture 2-3 $\mathrm{mL}$ peripheral blood was separated in an EDTA vacutainer, according to the recommendations for the most appropriate anticoagulant of Abend et al. [20], at precisely prescribed time intervals:

1) before beginning of RT

2) $24 \mathrm{~h}$ after the first, and before the second fraction of RT

3) $48 \mathrm{~h}$ after the first, and before the third fraction of RT

The adjuvant radiation treatment of all patients was carried out on linear accelerator Varian Clinac 23EX, with energy of photons of $15 \mathrm{MV}$, which is at the disposal at the University Clinic for Radiotherapy and Oncology in Skopje. The prescribed total tumor dose for every patient was $50 \mathrm{~Gy}$, divided in 25 fractions ( 5 weeks, with weekend brakes). The daily dose of $2 \mathrm{~Gy}$ was applied at every $24 \mathrm{~h}$ with dose-rate of $400 \mathrm{MU} / \mathrm{min}$. The elective RT after the performed CT (computerized tomography) simulation was conducted with 2 tangential and 1 supra/infraclavicular field of frontal thoracic wall and of regional lymphatic pool 
with 46 patients and with 2 tangential fields of frontal thoracic wall with 11 patients without supra/infraclavicular radiation.

\section{Total RNA Analysis \\ Processing of biological material for obtaining a sample (lysate of total leukocytes) for RNA isolation}

From the obtained 2-3 mL of EDTA-blood samples, a sample (lysate of total leukocytes) for RNA isolation was obtained. This was performed with selective lysis of the erythrocytes, i.e. with their osmotic lysis with solution for erythrocytes lysis. It was prepared ex tempore and represented solution of $\mathrm{NH}_{4} \mathrm{Cl}$ and $\mathrm{NH}_{4} \mathrm{HCO}_{3}$ in deionized water. More precisely, $300 \mu \mathrm{L}$ of the sample of EDTA blood and $1 \mathrm{~mL}$ of this solution was mixed for $10 \mathrm{~min}$ at room temperature following a 10-minute centrifugation and discarding of lysed erythrocytes. The remaining liquid mass from total leukocytes was immediately dissolved in $300 \mu \mathrm{L}$ RLT solution, which contained guanidine thiocyanate. Thus, lysate from total leukocytes was obtained, in which the present endogenous and, possibly exogenous ribonucleases, were inactivated from the strong chaotropic agent guanidine thiocyanate.

\section{Automated isolation of total RNA from lysate of total leukocytes}

The isolation procedure of total RNA was made through entirely automated process of Biorobot EZ1 and appropriate EZ1 RNA Tissue Mini Kits from the manufacturer Qiagen, Hilden, Germany at the Institute for Immunobiology and Human Genetics at the Faculty of Medicine in Skopje. The separation of the total RNA from the remaining cell components of this device are based on the application of magnetic particles in presence of chaotropic salt. According to manufacturer protocol, cartridge previously prepared lysed samples are placed on defined position of the reagent. The entire further process for isolation is automatic. The obtained isolate, as well as the lysate from leukocytes, was kept at a temperature of $-80^{\circ} \mathrm{C}$ until the next step - determination of expression of radioresponsive genes with qRT - PCR method.

\section{Gene expression analysis with qRT-PCR (quantitative reverse transcription polymerase chain reaction)}

The level of expression of the radioresponsive genes in peripheral blood from patients ionizied with RT was determined by $\Delta \Delta \mathrm{Ct}$ method, in the Laboratory for molecular pathology, Institute of Pathology, Faculty of Medicine in Skopje.

For reverse transcription we used High Capacity cDNA Archive Kit (Applied Biosystems, Foster City, CA, USA), according to the manufacturer's instructions. The temperature conditions for the process of reverse transcription were the following:

$$
\begin{aligned}
& 25^{\circ} \mathrm{C}-10 \mathrm{~min} \\
& 37^{\circ} \mathrm{C}-120 \mathrm{~min} \\
& 85^{\circ} \mathrm{C}-5 \mathrm{~min}
\end{aligned}
$$

After cDNAs were synthesized, qPCR on 7500 Real-Time PCR System (Applied Biosystems) was performed with TagMan Unversal Master Mix and primers used according to a study of Kbacik et al. [17].

The temperature conditions for the PCR were the following: 10 min initial denaturetion at $95^{\circ} \mathrm{C}$, followed by 40 cycles of $15 \mathrm{~s}$ denaturation at $95^{\circ} \mathrm{C}$ and 1 min annealing at $60^{\circ} \mathrm{C}$.

The following parameters were determined: $\Delta \Delta \mathrm{Ct}$ (FDXR-HPRT1) and $\Delta \Delta \mathrm{Ct}$ (CCNG1HPRT1), i.e., normalized expression of the target gene (FDXR, CCNG1) in relation to the endogen control, HPRT1; $2^{-\Delta \Delta \mathrm{Ct}}$ (FDXR-HPRT1) - fold change of the level of expression of 
the target gene FDXR in relation to HPRT1 and $2^{-\Delta \Delta C t}$ (CCNG1-HPRT1) - fold change of the level of expression of the target gene CCNG1 in relation to HPRT1.

Sequence of oligonucleotide primers that we used for Real Time PCR analysis are written in Table 1.

Table 1. Oligo sequence

\begin{tabular}{ll}
\hline \multicolumn{1}{c}{ Name of primer } & \multicolumn{1}{c}{ Oligo sequence 5' to 3' } \\
\hline HPRT1 F & TCAGGCAGTATAATCCAAAGATGGT \\
HPRT1 R & AGTCTGGCTTATATCCAACACTTCG \\
HPRT1 Probe & CGCAAGCTTGCTGGTGAAAAGGACCC \\
FDXR F & GTACAACGGGCTTCCTGAGA \\
FDXR R & CTCAGGTGGGGTCAGTAGGA \\
FDXR Probe & CGGGCCACGTCCAGAGCCA \\
CCNG1 F & GGAGCTGCAGTCTCTGTCAAG \\
CCNG1 R & TGACATCTAGACTCCTGTTCCAA \\
CCNG1 Probe & AACTGCTACACCAGCTGAATGCCC \\
\hline
\end{tabular}

\section{Statistical analysis}

The statistical analysis of the data obtained in this study was made with the statistical program SPSS 23.0. Kolmogorov-Smirnov test was used for testing of normality and distribution of data. For comparison of the analyzed variables at the three time points parametric and nonparametric tests for dependent samples (repeated-measures ANOVA, Friedman ANOVA, Wilcoxon Matched pairs, Cochran) were used.

The statistical significance was defined at the level of $p<0.05$.

\section{Results}

The statistical analysis as non-significant confirmed the differences in values of the threshold cycle of the host HPRT1 gene, in the analyzed period, before RT, 24 and 48 hours after the first fraction of RT $(\mathrm{p}=0.58)$. Gene expression levels of HPRT1 and differences between two periods of radiation are showed in Tables 2 and 3.

Table 2. HPRT1 Gene expression level in the analyzed period

\begin{tabular}{lcc}
\multicolumn{1}{c}{ Avrg Ct HPRT1 } & \multicolumn{2}{c}{ Descriptive statistics } \\
& mean \pm SD & median(IQR) \\
\hline Before first radiation & $31.23 \pm 2.3$ & $30.61(30.27-31.29)$ \\
24 h after first radiation & $31.13 \pm 2.4$ & $30.72(30.01-31.17)$ \\
48 h after first radiation & $31.27 \pm 2.2$ & $30.82(30.34-31.54)$ \\
\hline
\end{tabular}

Table 3. Differences of HPRT1 gene expression level between two periods of radiation

\begin{tabular}{|c|c|c|c|}
\hline \multicolumn{4}{|c|}{ Friedman ANOVA Sqr. $(\mathrm{N}=57, \mathrm{df}=\mathbf{2})=1.09 \mathrm{p}=\mathbf{0 . 5 8 1}$} \\
\hline \multicolumn{2}{|c|}{$\begin{array}{c}\text { Differences between periods of radiation } \\
\text { (Avrg Ct HPRT1) }\end{array}$} & $\mathbf{Z}$ & $p$ value \\
\hline Before first radiation & $24 \mathrm{~h}$ after first radiation & 1.498 & 0.134 \\
\hline Before first radiation & $48 \mathrm{~h}$ after first radiation & 0.163 & 0.871 \\
\hline $24 \mathrm{~h}$ after first radiation & $48 \mathrm{~h}$ after first radiation & 0.719 & 0.472 \\
\hline
\end{tabular}

A statistically significant difference in the threshold cycle for the gene CCNG1 $(p=0.014)$ was registered in the analyzed period. Post-hoc comparison in pairs showed that this total significance was due to a significantly lower value of the threshold cycle at the second time point in relation to the first $(\mathrm{p}=0.0009)$, and at the third in relation to the first time point ( $\mathrm{p}=0.028$ ), while the difference between the two time points after the applied RT was statistically insignificant $(\mathrm{p}=0.89)$ (Table 4 and 5). 
Table 4. CCNG1 Gene expression level in the analyzed period

\begin{tabular}{lcc}
\hline \multicolumn{1}{c}{ Avrg Ct CCNG1 } & \multicolumn{2}{c}{ Descriptive statistics } \\
& mean \pm SD & median(IQR) \\
\hline Before first radiation & $31.04 \pm 2.2$ & $30.44(29.91-31.36)$ \\
24 h after first radiation & $30.84 \pm 2.2$ & $30.34(29.64-31.15)$ \\
48 h after first radiation & $30.89 \pm 2.2$ & $30.21(29.84-30.98)$ \\
\hline
\end{tabular}

Table 5. Differences of CCNG1 gene expression level between two periods of radiation

\begin{tabular}{llcc}
\hline $\begin{array}{c}\text { Friedman ANOVA } \\
\text { Differences between periods of radiation } \\
\text { (Avrg Ct CCNG1) }\end{array}$ & $\mathbf{Z}$ & p value \\
\hline Before first radiation & 24 h after first radiation & 2.610 & 0.009 \\
Before first radiation & 48 h after first radiation & 2.197 & 0.028 \\
24 h after first radiation & 48 h after first radiation & 0.139 & 0.889 \\
\hline
\end{tabular}

The average values of the threshold cycle for CCNG1 were $31.04 \pm 2.2,30.84 \pm 2.2$ and $30.89 \pm 2.2$, respectively at the three time points. The median values amounted to 30.44 , 30.34 and 30.21 , respectively at the three time points.

The average and median values of the threshold cycle for FDXR were highest at the first time point $(33.71 \pm 2.7 ; 33.04)$ followed by the second $(33.19 \pm 2.8 ; 32.43)$ and the third time point $(32.95 \pm 2.6 ; 32.21)$ (Table 6$)$.

Table 6. FDXR Gene expression level in the analyzed period

\begin{tabular}{lcc}
\hline \multicolumn{1}{c}{ Avrg Ct FDXR } & \multicolumn{2}{c}{ Descriptive statistics } \\
& mean \pm SD & median(IQR) \\
\hline Before first radiation & $33.71 \pm 2.7$ & $33.04(32.39-33.62)$ \\
24 h after first radiation & $33.19 \pm 2.8$ & $32.43(31.74-33.24)$ \\
48 h after first radiation & $32.95 \pm 2.6$ & $32.21(31.81-32.75)$ \\
\hline
\end{tabular}

There was a statistically significant difference in the threshold cycle $(\mathrm{p}<0.0001)$ for FDXR in the analyzed period (before RT, 24 and 48 hours after the first fraction of RT). Significant differences were confirmed among all the three tested pairs $(p=0.000002$ for the difference between the second $v s$ the first time point ( $24 \mathrm{~h}$ after RT $v s$ the point before RT), $\mathrm{p}<0.0001$ for the difference between the third $v s$ the first time point (48h after RT $v s$ the point before RT), and $\mathrm{p}=0.0088$ for the difference between the third $v s$ the second time point ( $48 \mathrm{~h}$ after RT vs 24h after RT) (Table 7).

Table 7. Differences of FDXR gene expression level between two periods of radiation

\begin{tabular}{cccc}
\hline $\begin{array}{r}\text { Friedman ANOVA } \\
\text { Differences between periods of radiation } \\
\text { (Avrg Ct FDXR) }\end{array}$ & $\mathbf{Z}$ & p value \\
\hline Before first radiation & 24 $\mathrm{h}$ after first radiation & 4.763 & 0.000002 \\
Before first radiation & 48 h after first radiation & 5.216 & 0.000000 \\
24 h after first radiation & 48 h after first radiation & 2.618 & 0.0088 \\
\hline
\end{tabular}

The average values of the normalized expression of CCNG1 in relation to the "housekeeping" gene were $0.19 \pm 0.6$ at the first analyzed time point, $0.29 \pm 0.6$ at the second, $0.38 \pm 0.7$ at the third analyzed point. The differences in the average values of the parameter $\triangle \mathrm{Ct}$ (CCNG1-HPRT1) among the analyzed periods (before RT, 24 and 48 hours after the first fraction of RT) were not statistically significant $(\mathrm{p}=0.17)$ (Table 8$)$. 
Table 8. $\triangle \mathrm{Ct}$ values between CCNG1 and HPRT1 gene expressions

\begin{tabular}{lccc}
\hline$\Delta$ Ct $($ CCNG1-HPRT1) & \multicolumn{2}{c}{ Descriptive Statistics } & \\
$\mathbf{y}-\mathbf{a x i s}$ & mean \pm SD & min $-\mathbf{m a x}$ & Std.Error \\
\hline Before first radiation & $0.19 \pm 0.6$ & $-1.78-0.85$ & 0.081 \\
24 h after first radiation & $0.29 \pm 0.6$ & $-1.77-1.35$ & 0.081 \\
48 h after first radiation & $0.38 \pm 0.7$ & $-1.71-1.73$ & 0.089 \\
\hline $\mathrm{F}=1.8 ; \mathrm{P}=0.166$ & & &
\end{tabular}

The average values of the normalized expression of FDXR in relation to the "housekeeping" gene registered at the three analyzed time points amounted to $2.48 \pm 0.8$, $2.07 \pm 0.97$, and, $1.67 \pm 0.96$, consequently in the first, second and third time of taking blood (Table 9).

Table 9. $\Delta$ Ct values between FDXR and HPRT1 gene expressions

\begin{tabular}{lccc}
\hline \multicolumn{1}{c}{$\Delta$ Ct (FDXR-HPRT1) } & \multicolumn{3}{c}{ Descriptive Statistics } \\
\multicolumn{1}{c}{$\mathbf{y}$ - axis } & mean \pm SD & min - max & Std.Error \\
\hline Before first radiation & $2.48 \pm 0.8$ & $0.36-4.61$ & 0.117 \\
24 h after first radiation & $2.07 \pm 0.97$ & $0.12-5.63$ & 0.129 \\
48 h after first radiation & $1.67 \pm 0.96$ & $-0.61-3.93$ & 0.127 \\
\hline
\end{tabular}

The average values were significantly different at the three time points $(\mathrm{F}=30.3$ $\mathrm{p}<0.0001$ ) (Figure 1). Post-hoc comparison in pairs, using the correction of Bonferroni, confirmed significantly higher average value of $\Delta \mathrm{Ct}$ (FDXR-HPRT1) parameter before RT and 24 and 48 hours after its first application $(\mathrm{p}=0.01, \mathrm{p}<0.0001$, respectively) as well as between 24 and 48 hours after the first fraction of RT $(p<0.0001)$.

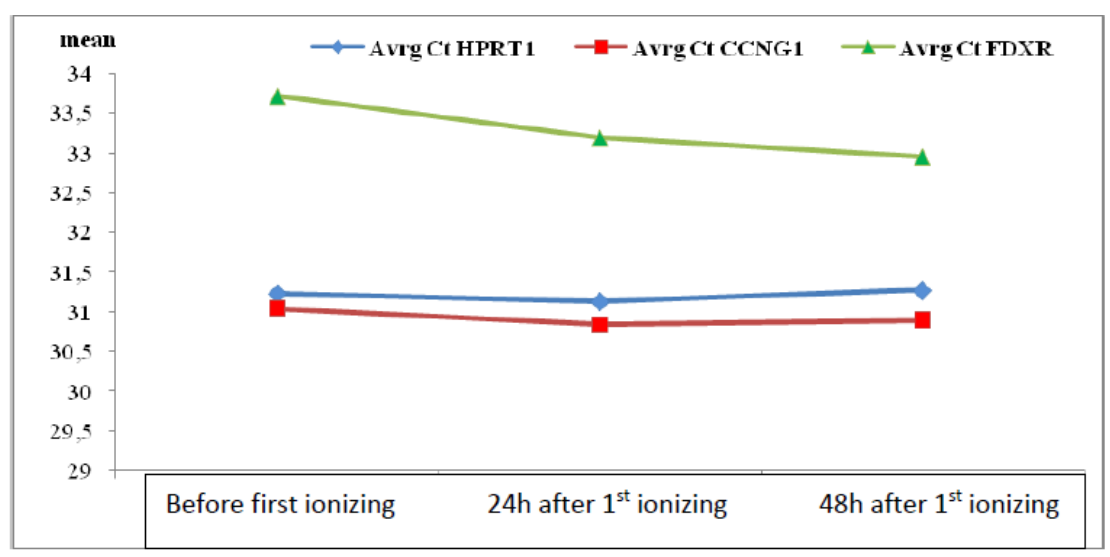

Fig. 1. Average $\mathrm{Ct}$ values of analyzed genes

Up-regulated gene expression of CCNG1 at the second in relation to the first time point, at the third in relation to the first time point, and at the third in relation to the second time point was registered in $35(61.4 \%), 38(66.8 \%)$ and $36(63.2 \%)$ patients, respectively.

The tested difference in distribution of patients with down- and up-regulated gene expression of CCNG1 at the analyzed time points was not statistically significant $(\mathrm{p}=0.8)$.

A statistically significant difference was confirmed when comparing patients with upregulated gene expression of FDXR at the analyzed time points $(p<0.04)$. According to the shown distribution of down- and up-regulated gene expression of FDXR at the analyzed time points, patients with up-regulated gene expression were registered more often at the third in relation to the first time point compared to the second in relation to the first, and the third in relation to the second time point - $50(87.7 \%)$ vs $47(82.5 \%)$ vs $41(71.9 \%)$, respectively. 


\section{Discussion}

Gene expression is emerging as a highly powerful readout for biodosimetry. Several studies demonstrated the applicability of microarray technology for analyzing large sets of transcripts for dose prediction for exposure. The analysis of a short list of genes, or even one gene by means of qPCR technology, is far more straightforward and cheaper compared to microarray analysis, while the accuracy of dose prediction is essentially similar [33].

Exposure to IR leads to many cellular responses including modification of the gene expression. Many genes have been reported to be radiation-responsive at the transcriptional level and monitoring of their expression in blood samples can potentially be used for rapid, minimally invasive high-throughput biological dosimetry purposes. Following a radiological accident, this would allow the identification of individuals exposed to high doses of radiation and requiring medical attention [22].

There is also a growing interest in low doses of radiation (typically below $100 \mathrm{mSv}$ ) and cellular responses as there is concern regarding the increasing use of IR in medical diagnostics and the long-term consequences of these exposures in terms of cancer induction. It has been recently reported that radiation exposure from CT scans in childhood with a cumulative dose of about $50 \mathrm{mGy}$ might almost triple the risk of leukemia.

Several studies have emphasized the need of application of IR based on changes of more genes [22,27,28], but recent data obtained from irradiated ex vivo and in vivo blood samples from patients suffering from prostate cancer subjected to RT suggest that FDXR represents the best stand-alone biomarker of exposure to IR [20]. A study published in 2018 [29] showed that there were no significant differences in expression of FDXR between ex vivo and in vivo samples of the same patients, which expression was significantly up-regulated $24 \mathrm{~h}$ after beginning of RT of certain organ in the majority of patients and remained increased during the whole period of the fractioned RT. Thereby, the potential confounding factors such as sex, infection and antioxidants only moderately affect the transcription of this gene. According to the study of Pallumbo et al. [30], the radiation-induced expression of this gene, in combination with that of other radioresponsive genes, may serve as a tool in following the individual response towards RT, taking a share in the development of personalized medicine.

FDXR encodes a mitochondrial flavoprotein that initiates electron transport for cytochrome $\mathrm{P} 450$ receiving electrons from NADPH. The gene is regulated by the p53 family and sensitizes cells to oxidative stress-induced apoptosis [34]. It is apparent that this pathway is particularly sensitive to radiation exposure and even more tightly and similarly regulated among different individuals compared to, for example. According to Abend et al., the cell cycle control through a gene such as CDKN1A, gene expression measurements comprises a higher interindividual variance than FDXR. This made FDXR the most promising candidate for highthroughput biological dosimetry [20].

In this study, we analyzed the transcriptional expression of 2 genes in 57 patients. In the analyzed period a statistical significant difference was registered in the threshold cycle for the gene CCNG1 $(p=0.014)$. Post-hoc comparison in pairs showed that this total significance was due to a significantly lower value of the threshold cycle at the second time point in relation to the first ( $\mathrm{p}=0.0009)$, and at the third in relation to the first time point $(\mathrm{p}=0.028)$, while the difference between the two time points after the applied RT was statistically insignificant $(\mathrm{p}=0.89)$.

For value of $\mathrm{p}<0.0001$, a statistically significant difference in the threshold cycle was confirmed for FDXR in the analyzed period (before RT, 24 and 48 hours after the first fraction of RT). Significant differences were confirmed among all three tested pairs ( $p=0.000002$ for the difference between the second $v s$ the first time point, $\mathrm{p}<0.0001$ for the difference between the third $v s$ the first time point, and $\mathrm{p}=0.0088$ for the difference between the third $v s$ the second time point). 
Modulation of gene expression is a major component of the cellular DNA damage response (DDR), a vital pathway for maintaining genomic stability and preventing cell death or cancer formation. Through p53 transcriptional target activation, the DDR also protects the genome from oxidation by endogenous reactive oxygen species (ROS). Importantly, our data support the concept that, even at very low doses, cells can detect external DNA insult and activate pathway.

Exposure to IR leads to complex cellular responses that include changes in gene expression, and these gene expression responses can differ between individuals. However, monitoring the expression level of these genes may be suitable for biological dosimetry. For these analyzed genes radiation responses are long-lasting and being up-regulated at $48 \mathrm{~h}$ postexposure. Confirmation and extension of these results in vivo with animal models or radiotherapy patient samples may enable the development of simple clinical tests to predict the likely level of radiation toxicity and to individualise patient treatment as well as to provide robust tools for biological dosimetry.

Conflict of interest statement. None declared.

\section{References}

1. Hall EJ, Giaccia AJ. Radiobiology for the radiologist, $8^{\text {th }}$ ed. Philadelphia: Wolters Kluwer; 2019.

2. Hall J, Jeggo PA, West C, Gomolka M, Quintens R, Badie C, et al. Ionizing radiation biomarkers in epidemiological studies - an update. Mutat Res Rev Mut Res 2017; 771: 59-84.

3. Димитровска А. Основи на радиотерапијата. Скопје: Медицински факултет, Универзитет „Св.Кирил и Методиј“; 2000.

4. Begley TJ, Samson LD. Network responses to DNA damaging agents. DNA Repair (Amst) 2004; 3(8-9): 1123-32.

5. Elkon R, Rashi-Elkeles S, Lerenthal Y, Linhart C, Tenne T, Amariglio N, et al. Dissection of a DNA-damage-induced transcriptional network using a combination of microarrays, RNA interference and computational promoter analysis. Genome Biol 2005; 6(5): R43.

6. Rashi-Elkeles S, Elkon R, Shavit S, Lerenthal Y, Linhart C, Kupershtein A, et al. Transcriptional modulation induced by ionizing radiation: p53 remains a central player. Mol Oncol 2011; 5(4): 336-48.

7. Innes CL, Hesse JE, Palii SS, Helmink BA, Holub AJ, Sleckman BP, et al. DNA damage activates a complex transcriptional response in murine lymphocytes that includes both physiological and cancer-predisposition programs. BMC Genomics 2013; 14: 163.

8. Kabacik S, Ortega-Molina A, Efeyan A, Finnon P, Bouffler S, Serrano M, et al. A minimally invasive assay for individual assessment of the ATM/CHEK2/p53 pathway activity. Cell Cycle 2011; 10: 1152-61.

9. Amundson SA, Grace MB, McLeland CB, Epperly MW, Yeager A, Zhan Q, et al. Human in vivo radiation-induced biomarkers: gene expression changes in radiotherapy patients. Cancer Res 2004; 64(18): 6368-71.

10. Kabacik S, Mackay A, Tamber N, Manning G, Finnon P, Paillier F, et al. Gene expression following ionising radiation: identification of biomarkers for dose estimation and prediction of individual response. Int J Radiat Biol 2011; 87(2): 115-29.

11. Paul S, Amundson SA. Development of gene expression signatures for practical radiation biodosimetry. Int J Radiat Oncol Biol Phys 2008; 71(4): 1236-44. 
12. Knops K, Boldt S, Wolkenhauer O, Kriehuber R. Gene expression in low- and highdose-irradiated human peripheral blood lymphocytes: possible applications for biodosimetry. Radiat Res 2012; 78(4): 304-12.

13. Chauhan V, Howland M, Wilkins R. Identification of gene-based responses in human blood cells exposed to alpha particle radiation. BMC Med Genomics 2014; 7: 43.

14. Lu TP, Hsu YY, Lai LC, Tsai MH, Chuang EY. Identification of gene expression biomarkers for predicting radiation exposure. Sci Rep 2014; 4: 6293.

15. Boldt S, Knops K, Kriehuber R, Wolkenhauer O. A frequency-based gene selection method to identify robust biomarkers for radiation dose prediction. Int J Radiat Biol 2012; 88(3): 267-76.

16. Dressman HK, Muramoto GG, Chao NJ, Meadows S, Marshall D, Ginsburg GS, et al. Gene expression signatures that predict radiation exposure in mice and humans. PLoS Med 2007; 4(4): 690-701.

17. Kabacik S, Mackay A, Tamber N, Manning G, Finnon P, Paillier F, et al. Gene expression following ionising radiation: identification of biomarkers for dose estimation and prediction of individual response. Int J Radiat Biol 2011; 87(2): 115129.

18. Lacombe J, Sima C, Amundson SA, Zenhausern F. Candidate gene biodosimetry markers of exposure to external ionizing radiation in human blood: A systematic review. PLOS One 2018; 13(6): e0198851.

19. Macaeva E, Saeys Y, Tabury K, Janssen A, Michaux A, Benotmane MA, et al. Radiation-induced alternative transcription and splicing events and their applicability to practical biodosimetry. Sci Rep 2016; 6: 19251.

20. Abend M, Badie C, Quintens R, Kriehuber R, Manning G, Macaeva E, et al. Examining Radiation-Induced In Vivo and In Vitro Gene Expression Changes of the Peripheral Blood in Different Laboratories for Biodosimetry Purposes: First RENEB Gene Expression Study. Radiat Res 2016; 185(2): 109-23.

21. Manning G, Macaeva E, Majewski M, Kriehuber R, Brzoska K, Abend M, et al. Comparable dose estimates of blinded whole blood samples are obtained independently of culture conditions and analytical approaches. Second RENEB gene expression study. Int J Radiat Biol 2017; 93(1): 87-98.

22. Manning G, Kabacik S, Finnon P, Bouffler S, Badie C. High and low dose responses of transcriptional biomarkers in ex vivo X-irradiated human blood. Int J Radiat Biol 2013; 89(7): 512-22.

23. Imamichi Y, Mizutani T, Ju Y, Matsumura T, Kawabe S, Kanno M, et al. Transcriptional regulation of human ferredoxin reductase through an intronic enhancer in steroidogenic cells. Biochim Biophys Acta 2014; 1839(1): 33-42.

24. Hwang PM, Bunz F, Yu J, Rago C, Chan TA, Murphy MP, et al. Ferredoxin reductase affects p53-dependent, 5-fluorouracil-induced apoptosis in colorectal cancer cells. Nat Med 2001; 7(10): 1111-7.

25. Liu G, Chen X. The ferredoxin reductase gene is regulated by the p53 family and sensitizes cells to oxidative stress-induced apoptosis. Oncogene 2002; 21(47): 7195-204.

26. Zhang Y, Qian Y, Zhang J, Yan W, Jung YS, Chen M, et al. Ferredoxin reductase is critical for p53-dependent tumor suppression via iron regulatory protein 2. Genes Dev 2017; 31(12): 1243-56.

27. Meadows S, Dressman H, Daher P, Himburg H, Russell JL, Doan P, et al. Diagnosis of partial body radiation exposure in mice using peripheral blood gene expression profiles. PLOS One 2010; 5(7): e11535.

28. Filiano AN, Fathallah-Shaykh HM, Fiveash J, Gage J, Cantor A, Kharbanda S, et al. Gene Expression Analysis in Radiotherapy Patients and C57BL/6 Mice as a Measure of Exposure to Ionizing Radiation. Radiat Res 2011; 176(1): 49-61. 
29. O’Brien G, Cruz-Garcia L, Majewski M, Grep J, Abend M, Port M, et al. FDXR is a biomarker of radiation exposure in vivo. Sci Rep 2018; 8: 684.

30. Palumbo E, Piotto C, Calura E, Fasanaro E, Groff E, Busato F, et al. Individual Radiosensitivity in Oncological Patients: Linking Adverse Normal Tissue Reactions and Genetic Features. Front Oncol 2019; 9: 987.

31. Tichy A, Kabacik S, O'Brien G, Pejchal J, Sinkorova Z, Kmochova A, et al. The first in vivo multiparametric comparison of different radiation exposure biomarkers in human blood. PLOS One 2018; 13: e0193412.

32. Cruz-Garcia L, O’Brien G, Donovan E, Gothard L, Boyle S, Laval A, et al. Influence of confounding factors on radiation dose estimation using in vivo validated transcriptional biomarkers. Health Phys 2018; 115(1): 90-101.

33. Macaeva E, Mysara MH, De Vos W, Baatout S, Quintens R. Gene expression-based biodosimetry for radiological incidents: assessment of dose and time after radiation exposure. Int J Radiat Biol 2019; 95(1): 64-75.

34. McLeland CB, Blakely WF. Real-time quantitative RT-PCR assay of GADD45 gene expression changes as a biomarker for radiation biodosimetry. Int J Radiat Biol 2002; 78: 1011-21. 